\title{
COMPACT AND WEAKLY COMPACT MULTIPLICATIONS ON C*-ALGEBRAS
}

\author{
Martin Mathieu*
}

Dedicated to Professor T. T. West on the occasion of his $50^{\text {th }}$ birthday.

\begin{abstract}
Let $\rho_{l}$ be a left and $\rho_{r}$ a right centralizer of a $\mathrm{C}^{*}$-algebra. We characterize when $\rho_{l} \rho_{r}$ is a compact or a weakly compact operator.
\end{abstract}

Following Vala [8], an element $a$ of a $C^{*}$-algebra $A$ is called compact if the mapping $x \mapsto a x a$ is a compact operator on $A$. For our purposes, the following equivalent definition due to Ylinen [10; Theorem 3.1] is more adequate: $a \in A$ is compact if and only if the left multiplication $L_{a}: x \mapsto a x$, or equivalently the right multiplication $R_{a}: x \mapsto x a$, is a weakly compact operator on $A$. Suppose for a moment that $A$ is the algebra $L(H)$ of all bounded operators on some Hilbert space $H$ and take $a, b \in A$, both non-zero. Vala had proved in [7] that $L_{a} R_{b}$ is compact if and only if both $a$ and $b$ are compact, whilst Akemann and Wright showed in [1; Proposition 2.3] that $L_{a} R_{b}$ is weakly compact if and only if either $a$ or $b$ is compact. This was extended to arbitrary prime $C^{*}$-algebras and $a, b$ in $M(A)$, the multiplier algebra of $A$, in [4], and similar results were obtained for linear combinations of left and right multiplications.

In the case of a general $\mathrm{C}^{*}$-algebra, Ylinen [9; Theorem 3.1] and also Akemann and Wright $[1 ; \mathrm{p} .146]$ proved that $L_{a} R_{a}$ is weakly compact if and only if $a$ is a compact element. In this short note we will characterize both $L_{a} R_{b}$ compact and $L_{a} R_{b}$ weakly compact for arbitrary $\mathrm{C}^{*}$-algebras. However, we will undertake this in the slightly more general framework of left and right centralizers. To this end, we first recall some facts from $[6 ; 3.12]$.

Let $A$ be a $C^{*}$-algebra. A linear map $\rho_{l}: A \rightarrow A$ having the property $\rho_{l}(x y)=$ $\rho_{l}(x) y$ for all $x, y \in A$ is called a left centralizer of $A$. Each left centralizer is bounded, and if we consider $A$ canonically embedded in its enveloping $\mathrm{W}^{*}$-algebra $A^{* *}$, then to each left centralizer $\rho_{l}$ of $A$ corresponds uniquely a left multiplier $a \in A^{* *}$, i.e., $\rho_{l}=L_{a}$ and $a A \subseteq A$. The norm closed subspace of all left multipliers of $A$ will be denoted by $\operatorname{LM}(A)$. The analogous concepts of right centralizer and right multiplier can be defined by $\rho_{r}=J \rho_{l} J$, where $J$ is the involution of $A$, and

* Research supported by Deutsche Forschungsgemeinschaft. 
$R M(A)=J(L M(A))$. Therefore, all compactness properties stated below for left centralizers apply equally well to right centralizers.

Let $K(A)$ denote the norm closed two-sided ideal of compact elements of $A$. We refer to [2] for some basic properties of $K(A)$. We first determine the weakly compact left centralizers of a $\mathrm{C}^{*}$-algebra.

Lemma 1. A left centralizer $\rho_{l}$ of $A$ is weakly compact if and only if $\rho_{l}=L_{a}$ for some $a \in K(A)$.

Proof. First observe that the second adjoint $L_{a}^{* *}$ of $L_{a}, a \in L M(A)$, is nothing but the mapping $x \mapsto a x, x \in A^{* *}$. If $L_{a}$ is weakly compact, $L_{a}^{* *} A^{* *} \subseteq A$ by [3; VI.4.2], which implies $a \in A$ and thus $a \in K(A)$ by the aforementioned characterization of compact elements [10; Theorem 3.1]. This proves the "only if"-part, and the "if"-part is clear. 口

Definition. Let $\rho_{l}=L_{a}$ and $\rho_{r}=R_{b}, a \in L M(A), b \in R M(A)$, be a left, respectively right, centralizer of a $C^{*}$-algebra $A$. The mapping $\rho_{l} \rho_{r}=L_{a} R_{b}$ is called a (two-sided) multiplication on $A$ and is denoted by $M_{a, b}$.

Suppose that either $a \in K(A)$ or $b \in K(A)$. Since the weakly compact operators form an ideal, $M_{a, b}$ is weakly compact. Suppose that both $a \in K(A)$ and $b \in K(A)$. Then $M_{a, b}$ is compact by virtue of the polarization identity

$$
M_{a, b}=\frac{1}{4} \sum_{k=0}^{3} i^{k} M_{\left(b+i^{k} a^{*}\right)^{*}, b+i^{k} a^{*}} .
$$

The converse implications do not hold for arbitrary $\mathrm{C}^{*}$-algebras. However, they are true for prime algebras. We prepare this by the following simple result.

Lemma 2. Let $A$ be a prime $C^{*}$-algebra and $a \in L M(A), b \in R M(A)$. Then $M_{a, b}=0$ if and only if $a=0$ or $b=0$.

Proof. If $M_{a, b}=0$, then $A a A A b A=0$. Thus $A a A$ and $A b A$ are orthogonal ideals of $A$ and the primeness of $A$ forces either $A a A=0$, i.e., $a=0$, or $A b A=0$, i.e., $b=0$. $\square$

Lemma 3. Let $A$ be a prime $C^{*}$-algebra and $a \in L M(A), b \in R M(A)$.

(i) If $M_{a, b}$ is weakly compact, $a \in K(A)$ or $b \in K(A)$.

(ii) If $M_{a, b} \neq 0$ is compact, $a \in K(A)$ and $b \in K(A)$.

The proof is very similar to that of the corresponding result in [4]. Therefore, we merely outline the argument for assertion (i). The weak compactness of $M_{a, b}$ implies $M_{a, b} A \subseteq K(A)$ since $M_{a x b, a x b}=M_{a, b} M_{x b, a x}$ is weakly compact for every $x \in A$. If $K(A)=0$, then $M_{a, b}=0$, and the assertion follows by Lemma 2. If $K(A) \neq 0$, we may assume that $A$ acts irreducibly on some Hilbert space $H$ and $K(A)=K(H)$, the compact operators on $H\left[2 ; \mathrm{C}^{*} .4\right.$ and F.4.3]. Thinking of $a$ 
and $b$ as left, respectively right, multipliers of $A$ in $A^{\prime \prime}=L(H)$, as we may do by [6; 3.12.3 and 3.12.5], it follows as in Lemma 1 that $M_{a, b} L(H) \subseteq K(H)$. Hence the induced multiplication on the Calkin algebra $C(H)=L(H) / K(H)$ vanishes, and since $C(H)$ is prime, either $a \in K(H)$ or $b \in K(H)$.

It is interesting to compare Lemma 3 with similar results in [5], where it is proved that the product $\delta_{1} \delta_{2}$ of two derivations of a prime $\mathrm{C}^{*}$-algebra is weakly compact if and only if either $\delta_{1}$ or $\delta_{2}$ is weakly compact [5; Lemma 4], and is compact if and only if both $\delta_{1}$ and $\delta_{2}$ are weakly compact and some additional condition holds [5; Lemma 7].

Before we can extend Lemma 3 to arbitrary $\mathrm{C}^{*}$-algebras we need another essentially well known result; for the sake of completeness we give a proof.

Lemma 4. Let $A$ be a $C^{*}$-algebra and $a \in L M(A), b \in R M(A)$. Then $M_{a, b}=0$ if and only if $a$ and $b$ are centrally orthogonal.

Proof. Denote by $z_{x}$ the central support projection of $x \in A^{* *}$. Since the set $\left\{x \in A^{* *} \mid x A^{* *} b=0\right\}$ is an ultraweakly closed ideal of $A^{* *}$ and contains $a$, it contains $z_{a}$. Similarly, the ideal $\left\{y \in A^{* *} \mid z_{a} A^{* *} y=0\right\}$ contains $z_{b}$. Therefore, $z_{a} z_{b}=0$, i.e., $a$ and $b$ are centrally orthogonal. This proves the "only if"-part, and the "if"-part is obvious. $\square$

We call $\rho_{l}=L_{a}$ and $\rho_{r}=R_{b}$ orthogonal if $a$ and $b$ are centrally orthogonal.

In what follows we assume that the $\mathrm{C}^{*}$-algebra $A$ acts in its reduced atomic representation on $H=\oplus_{t \in \hat{A}} H_{t}$, where $\hat{A}$ is the spectrum of $A$ (cf. $[6 ; 4.3 .7]$ ). Let $p_{t} \in A^{\prime}$ be the projection onto $H_{t} \hookrightarrow H, t \in \hat{A}$, and $p$ the central projection in $A^{* *}$ with $A^{* *} p=A^{\prime \prime}$. The multiplier algebra $M(A)$ of $A$ is the intersection $L M(A) \cap R M(A)$. If $T$ is a bounded linear map on a $\mathrm{C}^{*}$-subalgebra $B$ of $A^{* *}$ and $c \in M(B)$ is central, then $c T$ shall denote the map $x \mapsto c T(x)$ on $B$. Put $I_{l}=\overline{A \varrho_{l} A}$ and $I_{r}=\overline{A \varrho_{r} A}$. We can now characterize the (weakly) compact multiplications on $A$.

Theorem 1. Let $\rho_{l}$, respectively $\rho_{r}$, be a left, respectively right, centralizer of a $C^{*}$-algebra $A$. Then $\rho_{l} \rho_{r}$ is weakly compact if and only if there exist orthogonal central projections $e_{1}, e_{2}, e_{3}$ in $A^{* *}$ with $e_{1}+e_{2}+e_{3}=1$ and $a, b \in A^{* *}$, $c \in Z\left(A^{* *}\right)$ such that $c e_{i} \in M\left(I_{i}\right)$ where $I_{1}=I_{l} e_{1}, I_{2}=I_{r} e_{2}, i=1,2$, both $c \rho_{l \mid A e_{1}}=L_{a e_{1}}$ and $c \rho_{r \mid A e_{2}}=R_{b e_{2}}$ are weakly compact, $\rho_{r \mid A e_{1}}=R_{c b e_{1}}$ and $\rho_{l \mid A e_{2}}=L_{c a e_{2}}$, and $\rho_{l \mid A e_{3}}$ and $\rho_{r \mid A e_{3}}$ are orthogonal.

Proof. Let $\rho_{l}=L_{a_{0}}$ with $a_{0} \in L M(A)$ and $\rho_{r}=R_{b_{0}}$ with $b_{0} \in R M(A)$. Under the hypotheses on the projections $e_{j}$ and the elements $a, b$ and $c$, the identity

$$
\begin{aligned}
M_{a_{0}, b_{0}} & =M_{a_{0}, b_{0} e_{1}}+M_{a_{0} e_{2}, b_{0}}=M_{a_{0}, c b e_{1}}+M_{c a e_{2}, b_{0}} \\
& =M_{c a_{0} e_{1}, b}+M_{a, c b_{0} e_{2}}=M_{a e_{1}, b}+M_{a, b e_{2}}
\end{aligned}
$$

immediately proves the "if"-part. 
Suppose now that $M_{a_{0}, b_{0}}$ is weakly compact. Let $T_{0}$ be the set of those $t$ for which $M_{a_{0}, b_{0} \mid A p_{t}} \neq 0$. As in [1; Lemma 2.4] (see also [4; Lemma 3.5]), it follows that $T_{n}:=\left\{t \mid\left\|M_{a_{0}, b_{0} \mid A p_{t}}\right\|>1 / n\right\}$ is finite for each $n \in \mathbf{N}$ and thus $T_{0}=\bigcup_{n \in \mathrm{N}} T_{n}$ is countable. Put $e_{3}=\sum_{t \notin T_{0}} p_{t}+1-p$. Then $a_{0} e_{3}$ and $b_{0} e_{3}$ are centrally orthogonal (Lemma 4). Let $T_{0,1}$ be the set of those $t \in T_{0}$ such that $\rho_{l \mid A p_{t}}$ is weakly compact and $T_{0,2}=T_{0} \backslash T_{0,1}$. By Lemma $3, \rho_{r \mid A p_{t}}$ is weakly compact for all $t \in T_{0,2}$. Put $e_{1}=\sum_{t \in T_{0,1}} p_{t}, e_{2}=\sum_{t \in T_{0,2}} p_{t}, c=$ $\mathrm{c}\left(b_{0} b_{0}^{*}\right)^{\frac{1}{4}} e_{1}+\mathrm{c}\left(a_{0}^{*} a_{0}\right)^{\frac{1}{4}} e_{2}$, where $\mathrm{c}(y)$ denotes the central cover of a self-adjoint element $y \in A^{* *}[6 ; 2.6 .2]$, and $a_{1}=c a_{0} e_{1}, b_{2}=c b_{0} e_{2}$.

Take $t \in T_{0,1}$. Then

$$
\begin{aligned}
a_{1} p_{t} & =\mathrm{c}\left(b_{0} b_{0}^{*}\right)^{\frac{1}{4}} p_{t} a_{0}=\left\|\mathrm{c}\left(b_{0} b_{0}^{*}\right) p_{t}\right\|^{\frac{1}{4}} p_{t} a_{0} \\
& =\left\|\mathrm{c}\left(b_{0} b_{0}^{*} p_{t}\right)\right\|^{\frac{1}{4}} p_{t} a_{0}=\left\|b_{0} b_{0}^{*} p_{t}\right\|^{\frac{1}{4}} p_{t} a_{0}=\left\|b_{0} p_{t}\right\|^{\frac{1}{2}} p_{t} a_{0},
\end{aligned}
$$

where we used $[6 ; 2.6 .2$ and 2.6 .4$]$ and the fact that $p_{t}$ is a minimal projection in $Z\left(A^{* *}\right)$. Thus

$$
\left\|a_{1} p_{t}\right\|=\left\|b_{0} p_{t}\right\|^{\frac{1}{2}}\left\|a_{0} p_{t}\right\|=\left\|M_{a_{0} p_{t}, b_{0} p_{t}}\right\|^{\frac{1}{2}}\left\|a_{0} p_{t}\right\|^{\frac{1}{2}}
$$

tends to zero when $t$ runs through $T_{0,1}$ (recall that $T_{0,1} \cap T_{n}$ is finite for each $n \in \mathbf{N}$ ). We conclude that $c \rho_{l \mid A e_{1}}=L_{a_{1}}$ is the norm limit of a sequence of weakly compact operators and hence weakly compact itself [3; VI.4.4]. Similarly, $c \rho_{r \mid A e_{2}}=R_{b_{2}}$ is weakly compact.

Since $A p_{t} \cap K\left(H_{t}\right) \neq 0$, if $t \in T_{0}$ (it contains $\left.M_{a_{0}, b_{0}} A p_{t}\right), K\left(H_{t}\right)=K\left(A p_{t}\right) \subseteq$ $A p_{t}$ by $[6 ; 6.1 .4]$. Therefore, $\left(a_{1} p_{t}\right)_{t \in T_{0,1}}$ corresponds to a sequence $\left(a^{(n)}\right)_{n \in \mathrm{N}}$ in $A$ where each $a^{(n)}$ belongs to a closed ideal $I^{(n)}$ of $A, I^{(n)} \cong K\left(H_{t}\right)$ for a unique $t \in T_{0,1}, I^{(n)} \cap I^{(m)}=0$ if $n \neq m$, and $\lim _{n \rightarrow \infty}\left\|a^{(n)}\right\|=0$. By [4; Proposition 2.1], there is thus $a_{1}^{\prime} \in K(A)$ such that $a_{1}^{\prime} p_{t}=a_{1} p_{t}$ for every $t \in T_{0,1}$; so, in order to simplify the notation, we may assume that $a_{1}=a_{1}^{\prime} \in K(A)$ and similarly $b_{2} \in K(A)$. It follows that $c x a_{0} y e_{1}=x a_{1} y e_{1} \in A e_{1}$ for all $x, y \in A$; hence $c e_{1} \in M\left(I_{1}\right)$ and similarly $c e_{2} \in M\left(I_{2}\right)$.

Finally, observe that since $\left\|b_{0} p_{t}\right\|>0$ and \|\|$b_{0} p_{t}\left\|^{-\frac{1}{2}} b_{0} p_{t}\right\| \leq\left\|b_{0}\right\|^{\frac{1}{2}}$ for each $t \in T_{0,1}$, we may define

$$
b_{1}=b_{1} e_{1}=\sum_{t \in T_{0,1}} \oplus\left\|b_{0} p_{t}\right\|^{-\frac{1}{2}} b_{0} p_{t} \in A^{* *} e_{1} .
$$

Clearly, $c b_{1}=\sum_{t \in T_{0,1}}^{\oplus} \mathrm{c}\left(b_{0} b_{0}^{*}\right)^{\frac{1}{4}}\left\|b_{0} p_{t}\right\|^{-\frac{1}{2}} b_{0} p_{t}=b_{0} e_{1}$ and therefore $\rho_{r \mid A e_{1}}=R_{c b_{1}}$. Similarly, we put

$$
a_{2}=a_{2} e_{2}=\sum_{t \in T_{0,2}} \oplus\left\|a_{0} p_{t}\right\|^{-\frac{1}{2}} a_{0} p_{t} \in A^{* *} e_{2}
$$


and obtain $\rho_{l \mid A e_{2}}=L_{c a_{2}}$.

Hence we end up with $a=a_{1}+a_{2}+a_{0} e_{3}$ and $b=b_{1}+b_{2}+b_{0} e_{3}$, which completes the proof. $\square$

Theorem 2. Let $\rho_{l}$, respectively $\rho_{r}$, be a left, respectively right, centralizer of a $C^{*}$-algebra $A$. Then $\rho_{l} \rho_{r}$ is compact if and only if there exist $a, b$ in $K(A)$ such that $\rho_{l} \rho_{r}=M_{a, b}$. In addition, $a$ and $b$ can be chosen such that for some central projection $e$ in $A^{* *}$ and for some positive central multipliers $c$ and $d$ of $I_{l}(1-e)$ and $I_{r}(1-e)$, respectively, both $c \rho_{l \mid A(1-e)}=L_{d a(1-e)}$ and $d \rho_{r \mid A(1-e)}=R_{c b(1-e)}$ are weakly compact.

Proof. "If"-part: This follows from the remarks preceding Lemma 2.

"Only if"-part: Write $\rho_{l}=L_{a_{0}}, \rho_{r}=R_{b_{0}}$ with $a_{0} \in L M(A), b_{0} \in R M(A)$ as before, let $T_{0}$ be as in the proof of Theorem 1 and put $e=e_{3}$. Take $t \in T_{0}$. Since $\rho_{l \mid A p_{t}} \neq 0$ and $\rho_{r \mid A p_{t}} \neq 0$, Lemma 3 shows that both $a_{0} p_{t}$ and $b_{0} p_{t}$ have to be compact elements in $A p_{t}$. Putting

$$
a=\sum_{t \in T_{0}} \oplus\left\|a_{0} p_{t}\right\|^{-\frac{1}{2}}\left\|b_{0} p_{t}\right\|^{\frac{1}{2}} a_{0} p_{t} \quad \in A(1-e)
$$

and

$$
b=\sum_{t \in T_{0}}^{\oplus}\left\|a_{0} p_{t}\right\|^{\frac{1}{2}}\left\|b_{0} p_{t}\right\|^{-\frac{1}{2}} b_{0} p_{t} \quad \in A(1-e)
$$

yields $M_{a_{0}, b_{0}}=M_{a, b}$. Since $\left\|a p_{t}\right\|=\left\|b_{0} p_{t}\right\|^{\frac{1}{2}}\left\|a_{0} p_{t}\right\|^{\frac{1}{2}}=\left\|b p_{t}\right\|$ tends to zero when $t$ runs through $T_{0}$, both $a$ and $b$ are compact elements in $A$ by [4; Proposition 2.1] as in the proof of Theorem 1. Put $c=\mathrm{c}\left(b_{0} b_{0}^{*}\right)^{\frac{1}{4}}$ and $d:=\mathrm{c}\left(a_{0}^{*} a_{0}\right)^{\frac{1}{4}}$. The relations $c a_{0}(1-e)=d a(1-e)$ and $d b_{0}(1-e)=c b(1-e)$ are obviously valid; hence $c \rho_{l \mid A(1-e)}$ and $d \rho_{r \mid A(1-e)}$ are both weakly compact (Lemma 1). As in the proof of Theorem 1, we conclude that $c \in M\left(I_{l}(1-e)\right)$ and $d \in M\left(I_{r}(1-e)\right)$.

Corollary. Every compact multiplication on a $C^{*}$-algebra is the norm limit of multiplications of finite rank.

These results show that, apart from a direct summand where $\rho_{l} \rho_{r}$ can be zero, the (weak) compactness of $\rho_{l} \rho_{r}$ is completely determined by the weak compactness of $\rho_{l}$ and $\rho_{r}$ up to central 'scaling' factors. An extension of Theorems 1 and 2 to linear combinations of left and right centralizers (elementary operators) would first of all need a corresponding result as in Lemma 4, which is not available yet. 


\section{References}

[1] Akemann, C.A., and S. Wright: Compact actions on C*-algebras. - Glasgow Math. J. $21,1980,143-149$.

[2] Barnes, B.A., G.J. Murphy, M.R.F. Smyth, and T.T. West: Riesz and Fredholm theory in Banach algebras. - Pitman Research Notes in Mathematics 67, Boston, 1982.

[3] Dunford, N., and J.T. Schwartz: Linear operators, Part I. - Interscience, New York, 1958.

[4] Mathieu, M.: Elementary operators on prime C*-algebras, II. - Glasgow Math. J. 30, 1988, 275-284.

[5] Mathieu, M.: Properties of the product of two derivations of a C*-algebra. - Canad. Math. Bull., 1989 (to appear).

[6] Pedersen, G.K.: C*-algebras and their automorphism groups. - Academic Press, London, 1979.

[7] VAla, K.: On compact sets of compact operators. - Ann. Acad. Sci. Fenn. Ser. A I Math. 351, 1964.

[8] Vala, K.: Sur les éléments compacts d'une algèbre normée. - Ann. Acad. Sci. Fenn. Ser. A I Math. 407, 1967.

[9] Ylinen, K.: Dual C*-algebras, weakly semi-completely continuous elements, and the extreme rays of the positive cone. - Ann. Acad. Sci. Fenn. Ser. A I Math. 599, 1975.

[10] Ylinen, K.: Weakly completely continuous elements of $C^{*}$-algebras. - Proc. Amer. Math. Soc. 52, 1975, 323-326.

Universität Tübingen

Mathematisches Institut

Auf der Morgenstelle 10

D-7400 Tübingen

Federal Republic of Germany

Received 15 October 1987 